\title{
Tessier number 7 cleft with unilateral complete cleft lip and palate: a case report
}

\author{
Hyun Seung Lee ${ }^{1}$, Hyung Joon Seo ${ }^{1}$, Yong Chan Bae ${ }^{1,2}$ \\ ${ }^{1}$ Department of Plastic and Reconstructive Surgery, Pusan National University School of Medicine, Busan; ${ }^{2}$ Biomedical Research Institute, \\ Pusan National University Hospital, Busan, Korea
}

To date, there have been no reports of patients showing a Tessier number 7 cleft with unilateral complete cleft lip and palate. Furthermore, no studies have established the sequence, plan, or timing of surgical methods for treating patients presenting the above anomalies simultaneously. We report a case of a Tessier number 7 cleft with unilateral complete cleft lip and palate. Two months after birth, lip adhesion was performed on the unilateral complete cleft lip and total excision was performed on the skin tag. At 4 months of age, Tessier number 7 cleft was corrected. At 6 months of age, surgery involving two small triangular flaps was performed on the unilateral incomplete cleft lip after performing lip adhesion. At 13 months of age, two-flap palatoplasty with a vomer flap was performed on the complete cleft palate. At 6 years of age, open rhinoplasty was performed on the unilateral cleft lip nose deformity. At 9 years of age, bone grafting was performed for the alveolar cleft. At follow-up appointments up to 13 years of age, there were no major complications. Here, we present this patient, surgical procedures and timelines, and show our results demonstrating good postoperative outcomes.

Keywords Craniofacial abnormality / Orofacial cleft 7 macrostomia / Cleft lip / Surgical procedures, operative

\author{
Correspondence: Yong Chan Bae \\ Department of Plastic and \\ Reconstructive Surgery, Pusan \\ National University School of \\ Medicine, 179 Gudeok-ro, Seo-gu, \\ Busan 49241, Korea \\ Tel: +82-51-240-7273 \\ Fax: +82-51-243-9405 \\ E-mail: baeyc2@hanmail.net
}

This article was presented as a poster at the PRS KOREA 2020 conference on November 13-15, 2020.

Received: May 18, 2021 • Revised: July 17, 2021 • Accepted: August 6, 2021

pISSN: 2234-6163 • elSSN: 2234-6171 • https://doi.org/10.5999/aps.2021.00857 • Arch Plast Surg 2021;48:630-634

\section{INTRODUCTION}

Craniofacial cleft is an extremely rare disease, with a prevalence rate of approximately $0.24 \%$ in all reported cases of facial cleft [1]. Craniofacial cleft is a congenital disease that presents with facial disfigurement with multiple patterns and degrees of severity [2-4]. Tessier's classification, reported in 1976, has been the representative system for the classification of facial clefts. Tessier's classification is based on anatomical position; the different types of Tessier cleft are numbered from 0 to 14 , based on the central line which connects the sagittal plane and both orbital fossae [3].

Tessier number 7 craniofacial clefts, also referred to as temporozygomatic facial clefts or transverse facial clefts, are the most laterally located subtype of craniofacial clefts. Tessier number 7 is positioned on the line between the corner of the mouth and the ear [5]. This is a rare congenital anomaly, with a frequency of $1 / 60,000$ to $1 / 300,000$ normal births [6-8] and is characterized by soft-tissue abnormalities, such as macrostomia, external ear deformity, and hemifacial microsomia. Craniofacial clefts often involve the palate and lips [9]. However, to our knowledge, there have been no reports of patients showing the concomitant 
presence of cleft lip and palate and Tessier cleft 7. In addition, there are no studies to date establishing the sequence, plan, or timing of surgeries for treating patients presenting the above anomalies simultaneously. The aim of this article is to report this rare case and describe the staged management through several surgical procedures and the patient outcome. Written informed consent was obtained from the patient's parents for publication of this article and accompanying images. This study was approved by the institutional review board of our hospital (IRB No. 2009-029-095) and performed in accordance with the principles of the Declaration of Helsinki.

\section{CASE}

The patient was born via cesarean section in January 2006 at 39 weeks of gestation and had multiple anomalies at birth. There was no history of any relevant abnormalities in the family, and no teratogenic factors were identified clinically through history. He was diagnosed with esophageal atresia with tracheoesophageal fistula, left lateral cleft lip, left complete cleft lip and palate, skin tags on the right cheek and bilateral preauricular region, and right ocular limbal dermoid cyst (Fig. 1). Immediately after birth, he underwent genetic examination and was never diagnosed with specific syndrome or genetic abnormalities. Two days after the patient's birth, total correction was performed at the department of thoracic surgery for the treatment of esophageal atresia with tracheoesophageal fistula. Multiple surgical procedures were planned, including lip adhesion, correction of the lateral cleft lip, definite lip repair, cleft palate repair, interme-
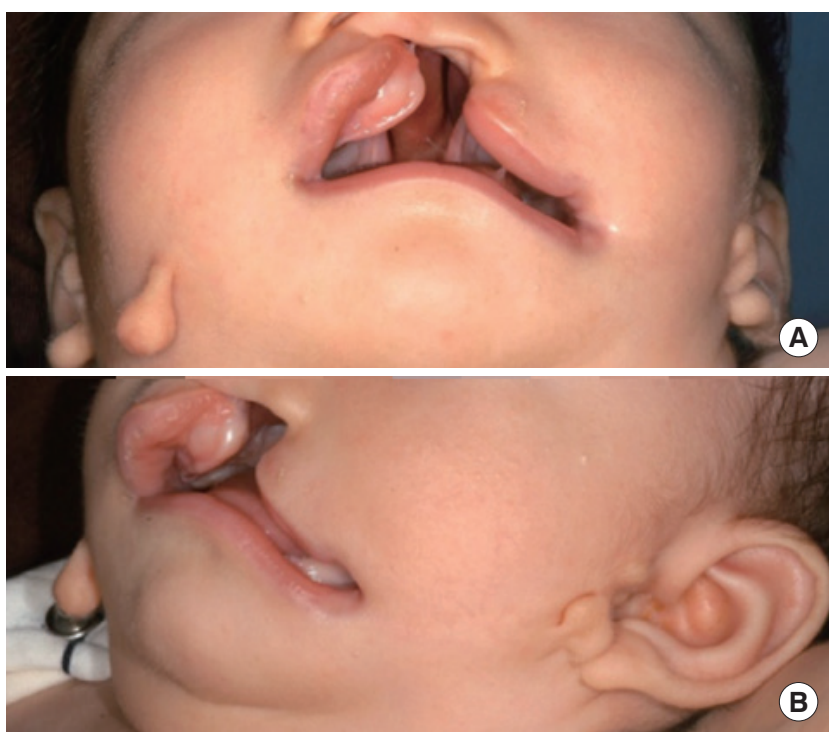

Fig. 1. Preoperative photographs at 2 months after birth. (A) Worm's eye view. (B) Oblique view. diate rhinoplasty, and repair of the alveolar cleft. Clinical and detailed radiological examination of the maxillofacial region was performed to assess the morphology of the cleft and plan treatment.

At 2 months of age, the patient underwent lip adhesion and total excision of the skin tags around the face. To preserve the anatomical landmark of the upper lip upon lip adhesion, a rectangular-shaped mucosal flap was elevated parallel to the vermilion-cutaneous junction (skin-vermilion ridge), at a distance of 2.0-3.0 $\mathrm{mm}$ from the apex of the Cupid's bow in each bilateral segment. A superiorly based vermilion mucosal flap was elevated in the lateral segment, while a buccal-based vermilion mucosal flap was elevated in the medial segment. A relaxing line was added to the skin and mucosa boundary at the base of the superiorly based vermilion mucosal flap, which was elevated in the lateral segments. The superiorly based vermilion mucosal flap was transpositioned and sutured to the mucosa of the relaxing line. The orbicularis oris muscle was repaired, the vermilion flap of the medial segment was sutured with the oral mucosa of the lateral segment, and skin closure was performed (Fig. 2).

At 4 months of age, correction for the left lateral cleft lip was performed. The procedure involved measurement of the distance between the apex of the Cupid's bow on the non-cleft side, the oral commissure on the non-cleft side, and the distance between the midpoint of the lower lip, defined as the midpoint between the central incisors and the oral commissure. Following the measurements, the point of symmetry in the left oral commissure (cleft side) was defined based on the distance to the expected location of the apex of the Cupid's bow upon definite lip repair and the distance to the midpoint of the lower lip, which were the same distance to the non-cleft side. Afterward, the expected oral commissural point was located $1 \mathrm{~mm}$ medially from the point of symmetry of the oral commissure. From the expected oral commissural point, a vertical line of incision in the vermilion was determined, and a cutaneous incision was made along the vermilion border toward the affected commissure. Following the incision, the orbicularis oris muscles were dis-

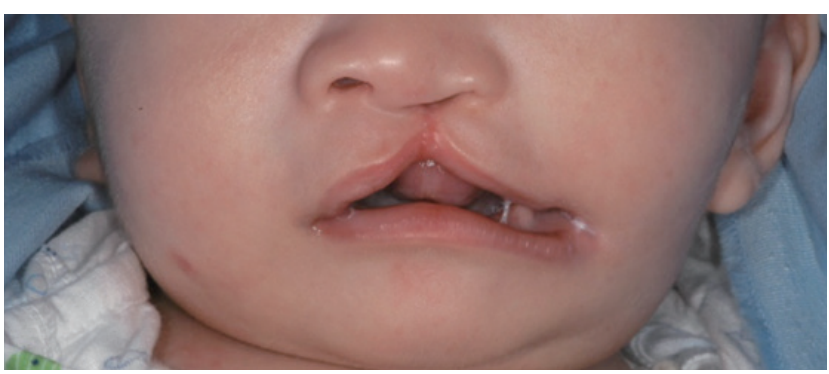

Fig. 2. Postoperative photo following lip adhesion at 4 months of age. 


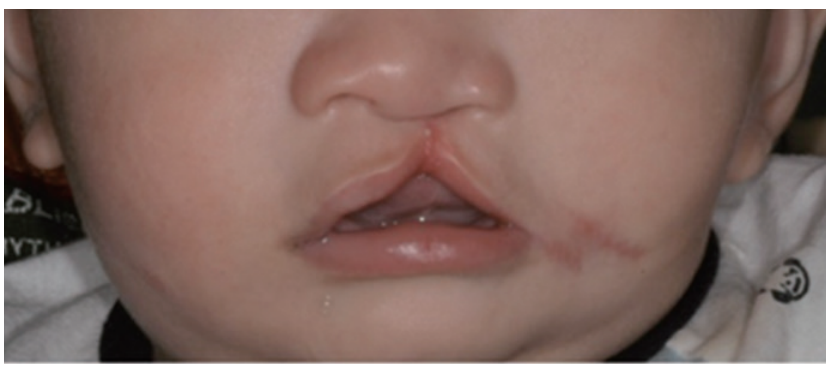

Fig. 3. Postoperative photo following correction of left lateral cleft lip at 6 months of age.

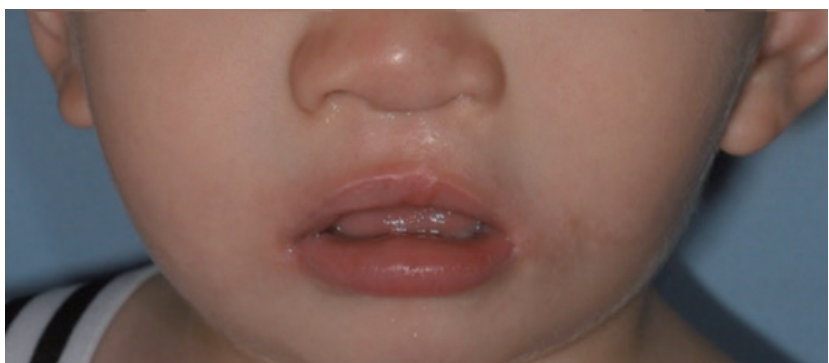

Fig. 4. Photograph of the patient following definite lip repair at approximately 1 year of age.

sected from the cutaneous and mucous layers. The orbicularis oris muscles of the upper and lower lips were approximated symmetrically, and muscle closure was performed using a horizontal mattress suture. Finally, the skin wound was trimmed, and Z-plasty was performed to situate the limb on the nasolabial fold (Fig. 3).

At 6 months of age, the patient underwent a definite lip repair for a unilateral incomplete cleft lip after lip adhesion was performed. Definite lip repair was performed using a straight-line closure with two small triangular flaps. After incision, supraperiosteal dissection was performed at the alar base of both segments to reduce tension. Next, the orbicularis oris muscle of each segment was repaired, and the oral mucosa was sutured, followed by trimming and repairing of the skin. To obtain symmetry in the depressed wide alar on the left side (cleft side) following lip repair, an infracartilaginous incision was made on the left alar rim. After incision, the soft tissue around the lower lateral cartilage was dissected to acquire freeing of the left lower lateral cartilage. A suspension suture was performed on the left lower lateral cartilage. Afterward, the remnant skin was trimmed and secured to obtain symmetry in the external naris (Fig. 4).

At 12 months of age, the left, complete, cleft palate was repaired using two-flap palatoplasties with a vomer flap. At 4 years of age, speech and language tests were performed by a speechlanguage pathologist. Upon evaluation, the patient's pronunciation and language ability were similar to those of peers of the

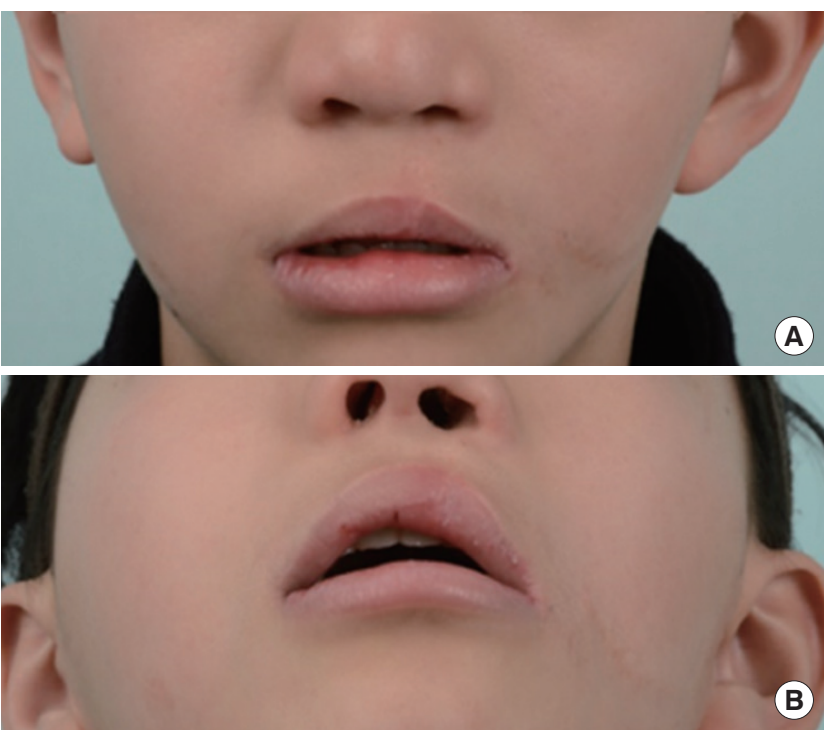

Fig. 5. Photographs of the patient at 13 years of age after the completion of all surgical procedures. (A) Frontal view. (B) Worm's eye view.

same age. At 5 years of age, the cleft lip nose deformity was corrected using open rhinoplasty. At 9 years of age, bone grafting was performed to reconstruct the left alveolar cleft. The patient was followed up until 13 years of age and showed only minor complications of surgical scarring and the need for orthodontic appliances. There were no major complications throughout his care (Fig. 5).

\section{DISCUSSION}

The rarity of the craniofacial cleft and the diverse presentation render developing a common diagnostic and treatment algorithm challenging [10]. The point of the oral commissure on the cleft side was needed for definite lip repair, and an apex of the Cupid's bow on the cleft side was needed to correct the lateral cleft lip. However, as some patients do not have such base points, it is important to determine the order of surgical correction. In this case, according to concerns regarding the distortion of the lip contour caused by the change in the position of the oral commissure by scar construction following lateral cleft lip repair, a definite lip repair was not immediately performed. Rather, lip adhesion was first performed on an area preserving the anatomical structure to transform the unilateral complete cleft lip into a unilateral incomplete cleft lip. Next, the apex of the Cupid's bow on the cleft side was estimated, and the lateral cleft lip was repaired. Following lateral cleft lip repair, correction of the apex of the Cupid's bow was performed by definite lip repair upon observing the change in position of the commissure due to scar construction. Afterward, other types of surgery, in- 
cluding palatopalsty, correction of cleft lip nose deformity, and repair of alveolar cleft, were performed in the usual order according to the schedule.

There are mixed suggestions for the appropriate timing for lip adhesion in the literature, ranging from 2 to 5 days after birth to 3.5 months of age [11-13]. In this case, the procedure was performed when the patient was 2 months old. This period allows for sufficient weight gain for the patient to safely undergo general anesthesia, to rule out concomitant malformation or disease, and for the parents to have enough time to prepare to take care of a child with a cleft lip. Although there are no clear recommendations on the appropriate timing of lateral cleft lip repair, research findings have suggested that an earlier procedure would be favorable for relieving anxiety among patients and caregivers. Some studies have recommended that lateral cleft lip repairs should be performed on patients $3-4$ months of age $[14,15]$. In this case, as lip adhesion was performed at 2 months of age and cleft lip repair was scheduled at 6 months of age, we planned correction of the lateral cleft lip at 4 months of age. These time points were judged more appropriate since they were consistent with the surgical periods of other studies $[14,15]$. Although the timing of definitive closure after lip adhesion remains controversial, closure was performed at approximately 6 months of age, similar to that observed in another study [11]. Palatoplasty for cleft palate repair was performed when the patient was 12 months old. The best speech results have been correlated with the closure of the palate close to the beginning of the infant's language acquisition, which for a normally developing child is before 12 months of age $[16,17]$. Due to a lack of symmetry of the external naris and alar collapse on the cleft side with growth, an intermediate rhinoplasty was performed before the child reached school age to minimize social stigma owing to a nasal deformity [18]. Although there are various methods of managing alveolar clefts, repair of the alveolar cleft was performed at a mixed dentition stage between 6 and 12 years of age.

Although no complications other than scarring at the surgical site and continuous orthodontic treatment were observed, the patient was placed under general anesthesia several times, which increases the number of times patients can be exposed to serious risk of respiratory and cardiac severe critical events [19]. However, it is difficult to correct facial deformities in a single surgical procedure, depending on the age of presentation and the maturation or growth pattern of facial structures, such as the nasal, palate, alveolus, and soft-tissue components. Several procedures are necessary to correct these defects $[5,20]$. Overall, despite undergoing multiple surgeries, the patient did not present with excessive surgical scarring and demonstrated normal pronunciation. Furthermore, the patient did not have any dis- turbance in the development of facial growth. Thus, it can be understood that complications due to the surgical procedures or technique were minimal.

Aside from procedures in the plastic surgery department, the patient underwent total correction of esophageal atresia with tracheoesophageal fistula at 2 days after birth in the department of thoracic and cardiovascular surgery. At 1 year of age, the patient underwent bilateral ear ventilation tube (V-tube) insertion in the department of otolaryngology for the treatment of serous otitis media, although recurrence was observed during followup. Thus, another bilateral V-tube insertion was performed when the patient was 5 years of age. The patient also underwent right partial keratectomy and lateral tarsorrhaphy in the department of ophthalmology at 2 years of age for the excision of a congenital ocular limbal dermoid cyst, although the cyst recurred.

Here, we presented the case of a patient demonstrating a simultaneous presence of cleft lip and palate, complete left and Tessier number $7 \mathrm{cleft}$, left. This rare case is difficult to repair due to the absence of anatomical landmarks on the lip on the cleft side, distortion of the nose and upper lip, distortion and deformity of the maxillary segments due to an alveolar cleft, and a wide cleft palate. Given that there have been no studies reporting details/protocols of surgeries for this rare phenomenon, this case may be helpful to surgeons working through a similar case in constructing a surgical plan, timing, and sequence.

\section{NOTES}

\section{Conflict of interest}

No potential conflict of interest relevant to this article was reported.

\section{Ethical approval}

The study was approved by the Institutional Review Board of Pusan National University Hospital (IRB No. 2009-029-095) and performed in accordance with the principles of the Declaration of Helsinki. Written informed consent was obtained.

\section{Patient consent}

The patient's parents provided written informed consent for the publication and the use of patient's images.

\section{Author contribution}

Conceptualization: YC Bae. Data curation: HS Lee. Formal analysis: HS Lee. Methodology: HS Lee, HJ Seo. Project administration: YC Bae. Visualization: HS Lee. Writing - original draft: HS Lee. Writing - review \& editing: YC Bae, HJ Seo. 


\section{ORCID}

Hyun Seung Lee https://orcid.org/0000-0002-8916-9325

Hyung Joon Seo https://orcid.org/0000-0003-1111-492X

Yong Chan Bae https://orcid.org/0000-0002-0268-4667

\section{REFERENCES}

1. Eppley BL, van Aalst JA, Robey A, et al. The spectrum of orofacial clefting. Plast Reconstr Surg 2005;115:101e-114e.

2. Kawamoto HK Jr. The kaleidoscopic world of rare craniofacial clefts: order out of chaos (Tessier classification). Clin Plast Surg 1976;3:529-72.

3. Tessier P. Anatomical classification facial, cranio-facial and latero-facial clefts. J Maxillofac Surg 1976;4:69-92.

4. Kawamoto HK Jr. Rare craniofacial clefts. In: McCarthy JG, editor. Plastic surgery. Philadelphia: Saunders; 1990. p. 292273.

5. Carstens MH. Pathologic anatomy of the soft palate, part 1 : embryology, the hard tissue platform, and evolution. J Cleft Lip Palate Craniofac Anomal 2017;4:37-64.

6. Kobraei EM, Lentz AK, Eberlin KR, et al. Macrostomia: a practical guide for plastic and reconstructive surgeons. J Craniofac Surg 2016;27:118-23.

7. Franco D, Franco T, da Silva Freitas R, et al. Commissuroplasty for macrostomia. J Craniofac Surg 2007; 18:691-4.

8. Fadeyibi IO, Ugburo AO, Fasawe AA, et al. Macrostomia: a study of 15 patients seen in Lagos, Nigeria and proposal for a classification of severity. J Plast Surg Hand Surg 2010;44: 289-95.

9. Gawrych E, Janiszewska-Olszowska J, Chojnacka H. Tessier type 3 oblique facial cleft with a contralateral complete cleft lip and palate. Int J Oral Maxillofac Surg 2010;39:1133-6.

10. Balaji SM. Modified canthopexy.J Maxillofac Oral Surg 2008; 7:414-9.

11. Millard DR Jr, Latham RA. Improved primary surgical and dental treatment of clefts. Plast Reconstr Surg 1990;86:85671.

12. Hamilton R, Graham WP 3rd, Randall P. The role of the lip adhesion procedure in cleft lip repair. Cleft Palate J 1971;8: $1-9$.

13. Cho B. Unilateral complete cleft lip and palate repair using lip adhesion and passive alveolar molding appliance. J Craniofac Surg 2001;12:148-56.

14. Koh SH, Jeong YW, Han JJ, et al. Orbicularis oris muscle reconstruction and cheiloplasty with Z-plasty in a patient with a transverse facial cleft. Maxillofac Plast Reconstr Surg 2019; 41:55.

15. Bordoloi U, Saikia R. Tessier cleft no. 7: report of 12 cases. J Evol Med Dent Sci 2014;3:6736-9.

16. Chapman KL, Hardin MA. Phonetic and phonologic skills of two-year-olds with cleft palate. Cleft Palate Craniofac J 1992; 29:435-43.

17. Dorf DS, Curtin JW. Early cleft palate repair and speech outcome. Plast Reconstr Surg 1982;70:74-81.

18. Shih CW, Sykes JM. Correction of the cleft-lip nasal deformity. Facial Plast Surg 2002;18:253-62.

19. Habre W, Disma N, Virag K, et al. Incidence of severe critical events in paediatric anaesthesia (APRICOT): a prospective multicentre observational study in 261 hospitals in Europe. Lancet Respir Med 2017;5:412-25.

20. Alonso N, Freitas RDS, de Oliveira E Cruz GA, et al. Tessier no. 4 facial cleft: evolution of surgical treatment in a large series of patients. Plast Reconstr Surg 2008;122:1505-13. 